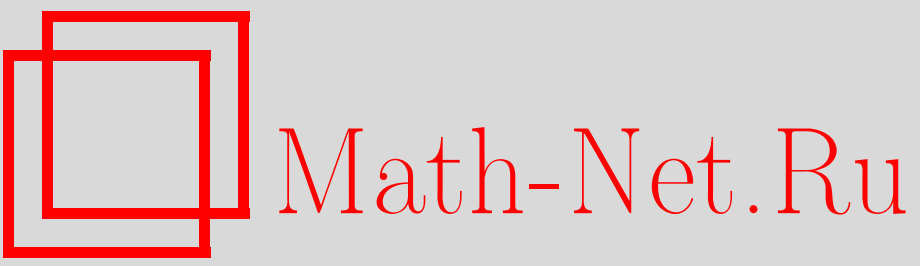

В. П. Павлов, Редукция на поверхность связей второго рода, ТМФ, 2002, том 132, номер 3, 399-407

DOI: https://doi.org/10.4213/tmf370

Использование Общероссийского математического портала Math-Net.Ru подразумевает, что вы прочитали и согласны с пользовательским соглашением

http://www.mathnet.ru/rus/agreement

Параметры загрузки:

IP : 44.207 .124 .84

26 апреля 2023 г., 17:23:04 
ТЕОРЕТИЧЕСКАЯ

И МАТЕМАТИЧЕСКАЯ

ФИЗИКА

Том 132, № 3

сентябрь, 2002

(C) 2002 г.

\author{
В.П. Павлов*
}

\title{
РЕДУКЦИЯ НА ПОВЕРХНОСТЬ СВЯЗЕЙ ВТОРОГО РОДА
}

Предложена рекуррентная процедура, позволяющая по данным связям второго рода явно строить эквивалентные им связи и каноническое преобразование так, что на поверхности связей скобка Дирака сводится к скобке Пуассона.

Ключевые слова: механика Гамильтона-Дирака, системы со связями, редукция на поверхность связей.

\section{1. ВВЕДЕНИЕ}

В работе [1] предложен вариант последовательной схемы перехода от лагранжева описания динамики систем с неголономными связями к гамильтонову описанию. В отличие от большинства исследований на эту тему, использовавших вслед за Дираком понятие "слабых" равенств (соотношений для классов эквивалентности), в этой работе все соотношения справедливы и вне поверхности связей, т.е. принят определенный способ выбора представителей классов эквивалентности. В данном подходе возникает процедура “размножения" связей, уточняющая схему Дирака и допускающая ее инвариантную формулировку [2]. С помощью этой процедуры удалось прояснить геометрический смысл связей второго рода и скобок Дирака, а также сформулировать стандартный прием, позволяющий найти все локальные симметрии модели с данной функцией Лагранжа [3] (см. также более раннюю работу [4]).

По поводу связей второго рода и скобки Дирака в работе [1] было упомянуто следующее, восходящее к работам [4], [5], утверждение. Неособым линейным преобразованием связей второго рода можно добиться того, чтобы матрища их скобок Пуассона приобрела нормальную форму (состояла бы из блоков $2 \times 2$ на главной диагонали, каждый из которых есть простейшая антисимметричная матрица $\left.\varepsilon_{i k}\right)$. После этого с помощью канонической замены переменных можно сделать преобразованные связи второго рода частью новых координат и импульсов. Тогда скобка Дирака сведется к скобке Пуассона в остальных новых канонических переменных. Ниже предлагается рекуррентная процедура, позволяющая явно реализовать указанное утверждение.

* Математический институт им. В. А. Стеклова РАН, Москва, Россия 


\section{2. РАЗМНОЖЕНИЕ СВЯЗЕЙ}

Поскольку рекурренция связана с самой процедурой размножения связей, напомним основные моменты этой процедуры. Мы начинаем с лагранжевой формы принципа Гамильтона, когда функциональными аргументами действия

$$
S[q]=\int_{t_{1}}^{t_{2}} d t L(q, \dot{q})
$$

являются обобщенные координаты $q=\left(q^{1}, q^{2}, \ldots, q^{n}\right)$. Чтобы воспользоваться техникой симплектической геометрии, следует модифицировать действие, включив в число его функциональных аргументов обобшенные скорости $v=\left(v^{1}, v^{2}, \ldots, v^{n}\right)$,

$$
S[q, v]=\int_{t_{1}}^{t_{2}} d t\left(L(q, v)-v^{i} \frac{\partial L}{\partial v^{i}}+\dot{q}^{i} \frac{\partial L}{\partial v^{i}}\right) .
$$

Однако в вырожденном случае, когда ранг гессиана $\Gamma_{i k}=\partial^{2} L / \partial v^{i} \partial v^{k}$ не максимален, одной этой модификации недостаточно - число функциональных аргументов действия приходится еще увеличить. Разобьем набор индексов $\{i\}=(1,2, \ldots, n)$ на два непересекающихся набора $\stackrel{0}{A}=\{a\}$ и $\stackrel{0}{M}=\{\mu\}=(1, \ldots, m)$ так, что $\Gamma_{a b}$-какой-нибудь минор максимального ранга. Тогда эквивалентные первоначальным уравнения движения получаются из условия экстремальности действия

$$
S[q, v, \breve{p}]=\int_{t_{1}}^{t_{2}} d t\left(L(q, v)-v^{a} \frac{\partial L}{\partial v^{a}}-p_{\mu} v^{\mu}+\dot{q}^{a} \frac{\partial L}{\partial v^{a}}+p_{\mu} \dot{q}^{\mu}\right),
$$

где $\breve{p}=\left(p_{1}, p_{2}, \ldots, p_{m}\right)$ - набор множителей Лагранжа, вариации по которым обеспечивают вьполнимость уравнений движения $\dot{q}^{\mu}=v^{\mu}$ и которые введены для компенсации вырожденности гессиана.

Переход к гамильтоновой форме динамики осушествляется следуюшим образом. Невырожденность гессиана $\Gamma_{a b}$ позволяет локально разрешить относительно $v^{a}$ определения импульсов $p_{a}=\partial L / \partial v^{a}$, получив $v^{a}=U^{a}(q, \hat{p}, \breve{v})$, где $\hat{p}$ - дополнительньй к $\breve{p}$ набор, а $\breve{v}=\left(v^{1}, v^{2}, \ldots, v^{m}\right)$. Обозначим символом \# подстановку $v^{a}=U^{a}$ для любой функции переменных $q, v, \breve{p}$. Благодаря матричному тождеству [6] $\Gamma_{\mu \nu}=\Gamma_{\mu \alpha} \Gamma^{a b} \Gamma_{b \nu}$ (матрица с индексами вверху обратна к $\Gamma_{a b}$ ), функция Рауса

$$
R(q, \hat{p}, \breve{v})=\left(L-v^{a} \frac{\partial L}{\partial v^{a}}\right)^{\#}
$$

линейна по $\breve{v}$,

$$
R(q, \hat{p}, \breve{v})=-H(q, \hat{p})+v^{\mu} \psi_{\mu}(q, \hat{p}),
$$

причем $\psi_{\mu}(q, \hat{p})=\left(\partial L / \partial v^{\mu}\right) \#$. Введем обозначение

$$
\varphi_{\mu}=p_{\mu}-\psi_{\mu} .
$$


В результате получаем, что замена функциональных аргументов $\hat{v} \rightarrow \hat{p}$ приводит действие (1) к гамильтоновой форме

$$
S[q, p, \breve{v}]=\int_{t_{1}}^{t_{2}} d t\left(-H-v^{\mu} \varphi_{\mu}+p_{i} \dot{q}^{i}\right),
$$

а уравнения Эйлера-Лагранжа для него принимают предложенный Дираком вид

$$
\begin{aligned}
\dot{q}^{i} & =\left\{H, q^{i}\right\}+v^{\nu}\left\{\varphi_{\nu}, q^{i}\right\}, \\
\dot{p}_{i} & =\left\{H, p_{i}\right\}+v^{\nu}\left\{\varphi_{\nu}, p_{i}\right\}, \\
\varphi_{\mu} & =0 .
\end{aligned}
$$

Здесь $\{\cdot, \cdot\}$ - стандартная скобка Пуассона в переменных $p, q$. Отметим, что в качестве множителей Лагранжа для действия (4) выступают скорости $\breve{v}$, а для действия (1) импульсы $\breve{p}$. Поэтому в обоих случаях разумно включить их в число локальных координат фазового пространства. При этом в гамильтоновом случае производная по времени от любой функции, заданной на этом пространстве, в силу уравнений движения примет вид

$$
\dot{f}=\{H, f\}+v^{\nu}\left\{\varphi_{\nu}, f\right\}+\dot{v}^{\nu} \frac{\partial f}{\partial v^{\nu}} .
$$

Согласно Дираку условие совместности системы уравнений (5) означает, что производная по времени в силу уравнений движения для (первичных) связей $\varphi_{\mu}$ должна сводиться к линейной комбинации связей с некоторыми функциями на фазовом пространстве в качестве коэффициентов. В соответствии с формулой (6) получим

$$
\dot{\varphi}_{\mu}=\left\{H, \varphi_{\mu}\right\}+v^{\nu}\left\{\varphi_{\nu}, \varphi_{\mu}\right\}
$$

По определению (3) функции $\varphi_{\mu}$ линейны по $p_{\mu}$ с единичным коэффициентом, поэтому оба члена правой части соотношения (7) не зависят от $p_{\mu}$, упомянутая линейная комбинация равна нулю и для выполнения условия совместности мы должны потребовать, чтобы $\dot{\varphi}_{\mu}=0$.

Рассмотрим матрицу $\gamma_{\nu \mu}=\left\{\varphi_{\nu}, \varphi_{\mu}\right\}$. Если ее ранг на поверхности первичных связей $M_{0}=\left\{\varphi_{\mu}=0, \mu \in \stackrel{0}{M}\right\}$ не максимален, выделим какой-нибудь минор наибольшего ранга $\gamma_{\alpha \beta}$, разбив набор индексов $\stackrel{0}{M}$ на два непересекаюшихся набора $\stackrel{1}{A}=\{\alpha\}$ и $\stackrel{1}{M}=\left\{\mu^{\prime}\right\}$. Связи $\varphi_{\alpha}$ называются (первичными) связями второго рода. При этом на поверхности $M_{0}$ имеем $\gamma_{\mu^{\prime} \nu^{\prime}}=\gamma_{\mu^{\prime} \alpha} \gamma^{\alpha \beta} \gamma_{\beta \gamma^{\prime}}$, а в силу линейности функций $\varphi_{\mu}$ по $p_{\mu}$ это будет справедливо и вне $M_{0}$. Тогда в качестве нулевых векторов матришы $\gamma_{\mu \nu}$ можно выбрать

$$
\beta_{\mu^{\prime}}^{\mu}=\delta_{\mu^{\prime}}^{\mu}-\delta_{\alpha}^{\mu} \gamma^{\alpha \beta} \gamma_{\beta \mu^{\prime}}
$$

Требование $\dot{\varphi}_{\alpha}=0$ позволяет фиксировать множители Лагранжа $v^{\alpha}=-\left(\left\{H, \varphi_{\beta}\right\}+\right.$ $\left.v^{\mu^{\prime}} \gamma_{\mu^{\prime} \beta}\right) \gamma^{\beta \alpha}$, так что производная по времени в силу уравнений движения примет вид

$$
\dot{f}=\{H, f\}_{1}^{*}+v^{\nu^{\prime}}\left\{\varphi_{\nu^{\prime}}, f\right\}_{1}^{*}+\dot{v}^{\nu} \frac{\partial f}{\partial v^{\nu}}
$$

3 Теоретическая и математическая физика, т. 132, № 3, 2002 г. 
где $\{\cdot, \cdot\}_{1}^{*}-$ скобка Дирака $\{\cdot, \cdot\}^{\mathrm{D}}$, построенная по первичным связям второго рода,

$$
\{f, g\}_{1}^{*}=\{f, g\}-\left\{f, \varphi_{\alpha}\right\} \gamma^{\alpha \beta}\left\{\varphi_{\beta}, g\right\} .
$$

Строго говоря, в последнем члене формулы (9) следовало бы подставить вместо $\dot{v}^{\alpha}$ их значения, вычисленные с помощью первоначального уравнения (6). Поскольку для дальнейших рассуждений не важен довольно громоздкий явный вид этих выражений, мы их не выписываем, заметив только, что член с ускорениями примет вид $\dot{v}^{\nu^{\prime}} \beta_{\nu^{\prime}}^{\nu} \partial f / \partial v^{\nu}$.

Если матрица $\gamma_{\nu \mu}$ невырож денна (набор $M$ пуст), все множители Лагранжа окажутся фиксированными, а условие совместности выполненным. В противном случае умножение условия $\dot{\varphi}_{\mu}=0$ на нулевые векторы матрицы $\gamma_{\nu \mu}$ дает вторичные связи,

$$
0=\left\{H, \varphi_{\mu}\right\} \beta_{\mu^{\prime}}^{\mu}=\left\{H, \varphi_{\mu^{\prime}}\right\}_{1}^{*} \equiv \stackrel{1}{\varphi}_{\mu^{\prime}}
$$

Требование совместности сужает при этом поверхность связей до

$$
M_{1}=\left\{\varphi_{\mu}=0, \mu \in \stackrel{0}{M} ; \stackrel{1}{\varphi}_{\mu^{\prime}}=0, \mu^{\prime} \in \stackrel{1}{M}\right\} .
$$

\section{3. ПРИВЕДЕНИЕ МАТРИЦЫ $\gamma_{\alpha \beta}$ К НОРМАЛЬНОЙ ФОРМЕ}

Возьмем какой-нибудь ненулевой элемент матрицы $\gamma_{\alpha \beta}$ и временно обозначим $\varphi_{\alpha}=$ $\xi, \varphi_{\beta}=\eta,\{\xi, \eta\}=\gamma_{0}^{-1}$. Очевидно, $\left\{\xi, \gamma_{0} \eta\right\}=1+\left\{\xi, \gamma_{0}\right\} \eta$; обозначив $\gamma_{i}=\left\{\xi, \gamma_{0} \gamma_{i-1}\right\}$, построим формальньй ряд

$$
\begin{aligned}
\tilde{\eta} & =\gamma_{0}\left(\eta-\frac{\gamma_{1} \eta^{2}}{2 !}+\frac{\gamma_{2} \eta^{3}}{3 !}-\ldots\right)= \\
& =\gamma_{0} \int_{0}^{\eta} d \eta\left(1-\gamma_{1} \eta+\frac{\gamma_{2} \eta^{2}}{2 !}-\frac{\gamma_{3} \eta^{3}}{3 !}+\ldots\right)=\gamma_{0} \int_{0}^{\eta} d \eta \exp \left(-\eta\left\{\xi, \gamma_{0} \cdot\right\}\right) \circ 1 .
\end{aligned}
$$

Последний интеграл следует понимать как формальньй псевдодифференциальный оператор, вспомнив, что взятие скобки Пуассона связи $\varphi$ с функцией $f(p, q)$ эквивалентно действию на эту функцию векторного поля $X_{d \varphi}$, соответствуюшего в симплектической геометрии дифференциалу связи. В этих обозначениях

$$
\tilde{\eta}=\gamma_{0} \int_{0}^{\eta} d \eta \exp \left(-\eta X_{d \xi} \gamma_{0}\right) \circ 1 \equiv X^{(\xi)} \eta,
$$

где $X^{(\xi)}$ - упомянутый псевдодифференциальный оператор. Очевидно, что коэффициенты ряда (12) подобраны так, что $\{\xi, \tilde{\eta}\}=1$, а преобразование $(\xi, \eta) \rightarrow(\xi, \tilde{\eta})$ является неособым.

Пусть теперь $\{\xi, \eta\}=1$, а $\zeta$ - любая из остальных связей второго рода. Обозначив $\left(X_{d \xi}\right)^{n} \zeta=\delta_{n},\left(X_{d \eta}\right)^{n} \zeta=\varepsilon_{n}$, построим аналогичные $(12)$ формальные ряды

$$
\begin{aligned}
& \tilde{\zeta}=\zeta-\frac{\delta_{1} \eta}{1 !}+\frac{\delta_{2} \eta^{2}}{2 !}-\frac{\delta_{3} \eta^{3}}{3 !}+\ldots=\exp \left(-\eta X_{d \xi}\right) \zeta \equiv Y^{(\xi)} \zeta, \\
& \tilde{\tilde{\zeta}}=\zeta+\frac{\varepsilon_{1} \xi}{1 !}+\frac{\varepsilon_{2} \xi^{2}}{2 !}+\frac{\varepsilon_{3} \xi^{3}}{3 !}+\ldots=\exp \left(\xi X_{d \eta}\right) \zeta \equiv Y^{(-\eta)} \zeta .
\end{aligned}
$$


Их структура обеспечивает выполнимость условий $\{\xi, \tilde{\zeta}\}=0=\{\eta, \tilde{\zeta}\}$, однако нам нужно одновременное обрашение в нуль скобок Пуассона преобразованной связи $\zeta$ с обеими связями $\xi, \eta$. Это достигается за счет того, что операции $Y^{(\xi)}$ и $Y^{(-\eta)}$ коммутируют, поскольку перестройка выражения $Y^{(\xi)} Y^{(-\eta)} \zeta$ в $Y^{(-\eta)} Y^{(\xi)} \zeta$ осушествляется за счет тождества Якоби $X_{d \xi} X_{d \eta} \zeta=X_{d \eta} X_{d \xi} \zeta+X_{d \zeta}\{\xi, \eta\}$, где второй член в правой части обрашается в нуль вследствие равенства $\{\xi, \eta\}=1$ (подробнее см. приложение). Итак, выражение $\zeta^{*}=Y^{(\xi)} Y^{(-\eta)} \zeta$ имеет нулевую скобку Пуассона с обеими связями $\xi, \eta$. Выполним такой переход для всех остальных связей второго рода. Новая матрица $\gamma_{\alpha \beta}$ будет иметь матрицу $\varepsilon_{i k}$ в левом верхнем углу, а остальные элементы первых двух строк и столбцов будут нулевыми. В приложении (см. формулу (П.3)) показано, что эта новая матрица останется невырожденной, а структура разложений (12) и (14) обеспечит эквивалентность новых связей старым. Теперь можно взять следуюший ненулевой элемент из правого нижнего блока и повторить процедуру, что завершает рекурренцию.

\section{4. КАНОНИЧЕСКОЕ ПРЕОБРАЗОВАНИЕ}

Описанный в разделе 2 переход к гамильтоновой форме динамики таков, что при любом выборе локальных координат первичные связи (3) линейны по импульсам: часть импульсов оказывается "маркированной". Назвав преобразованные по процедуре раздела 3 связи новыми координатами и импульсами, мы должны "подправить" остальные координаты и импульсы. Возьмем какую-нибудь их пару $p, q$. Прежде всего, необходимо обеспечить равенство нулю скобок Пуассона этих $p, q$ с преобразованными связями. Очевидно, что это достигается действием на них пар псевдодифференциальных операторов $Y^{(\xi)}$ и $Y^{(-\eta)}$, отвечающих всем преобразованным связям второго рода. В приложении (см. формулу (П.6)) показано, что скобка Пуассона преобразованных таким образом $p, q$ выражается через скобку Дирака, построенную по преобразованным связям,

$$
\left\{Y^{(\xi)} Y^{(-\eta)} p, Y^{(\xi)} Y^{(-\eta)} q\right\}=Y^{(\xi)} Y^{(-\eta)}\{p, q\}_{\xi, \eta}^{\mathrm{D}},
$$

а она, вообще говоря, не единична. Поэтому для преобразованных только что $p, q$ необходимо дополнительное преобразование типа (12). Поскольку же правая часть (15) обрашается в нуль под действием $X_{d \xi}$ и $X_{d \eta}$, скобки Пуассона дополнительно преобразованных $p, q$ с новыми связями второго рода останутся нулевыми.

В сушности, процедура "подправки" новых координат и импульсов повторяет процедуру приведения матрицы $\gamma_{\alpha \beta}$ к нормальному виду. После ее завершения мы приходим к уравнению движения

$$
\dot{f}=\{H, f\}^{M_{\alpha}}+v^{\nu^{\prime}}\left\{\varphi_{\nu^{\prime}}, f\right\}^{M_{\alpha}}+\dot{v}^{\nu^{\prime}} \beta_{\nu^{\prime}}^{\nu} \frac{\partial f}{\partial v^{\nu}},
$$

где символом $\{\cdot, \cdot\}^{M_{\alpha}}$ обозначена скобка Пуассона, суженная на поверхность первичных связей второго рода $M_{\alpha}=\left\{\varphi_{\alpha}=0\right\}$, и к суженным на эту поверхность уравнениям связей $\varphi_{\mu^{\prime}}=0$. Условие совместности для этой системы приводит к уравнениям вторичных связей $\stackrel{1}{\varphi}_{\mu^{\prime}} \equiv\left\{H, \varphi_{\mu^{\prime}}\right\}^{M_{\alpha}}=0$. При этом матрица суженных скобок Пуассона 
для первичных связей $\varphi_{\mu}$ является просто нулевой: преобразовав эти связи с помощью формул (14), мы всюду получим $\gamma_{\alpha \mu^{\prime}}=0$. Вследствие матричного тождества то же будет справедливо для $\gamma_{\nu^{\prime} \mu^{\prime}}$, а ряды $(14)$ на поверхности $M_{\alpha}$ сводятся к первым членам, т.е. самим связям $\varphi_{\mu^{\prime}}$.

Далее необходимо обеспечить согласованность уравнений (16) и уравнений вторичных связей. Мы приходим к матрице (суженных на поверхность $M_{\alpha}$ ) скобок Пуассона $\stackrel{1}{\gamma}_{\nu^{\prime} \mu^{\prime}}=\left\{\varphi_{\nu^{\prime}}, \stackrel{1}{\varphi}_{\mu^{\prime}}\right\}^{M_{\alpha}}$ и, если ее ранг ненулевой, повторяем процедуру раздела 3.

В реальном проведении рекуррентной процедуры встречаются некоторые особенности. В качестве примера рассмотрим случай, когда имеется только одна первичная связь.

\section{5. ПРИМЕР: $\sigma$-МОДЕЛЬ}

Зададим $\sigma$-модель функцией Лагранжа

$$
L(\mathbf{n}, \dot{\mathbf{n}}, \sigma)=2^{-1}\left(\dot{\mathbf{n}}^{2}+\sigma\left(\mathbf{n}^{2}-\gamma\right)\right)
$$

где обобщенными координатами являются вектор $\mathbf{n} \subset R^{n}$ и скаляр $\sigma \subset R^{1}$, а постоянная $\gamma>0$. Модифицированное лагранжево действие имеет вид

$$
S[\mathbf{n}, \sigma ; \mathbf{v}, \nu ; \pi]=\int_{t_{1}}^{t_{2}} d t\left(L(\mathbf{n}, \mathbf{v}, \sigma)-\mathbf{v} \frac{\partial L}{\partial \mathbf{v}}-\pi \nu+\dot{\mathbf{n}} \frac{\partial L}{\partial \mathbf{v}}+\pi \dot{\sigma}\right) .
$$

Гессиан однократно вырожден (не зависит от скорости $\nu$ ); формула, определяюшая импульсы $\mathbf{p}=\partial L / \partial \mathbf{v}$, элементарно разрешается относительно обобшенных скоростей $\mathbf{v}$, и мы приходим к гамильтонову действию

$$
S[\mathbf{n}, \sigma ; \mathbf{p}, \pi ; \nu]=\int_{t_{1}}^{t_{2}} d t\left(-h-\nu \varphi_{0}+\mathbf{p} \dot{\mathbf{n}}+\pi \dot{\sigma}\right)
$$

с функцией Гамильтона

$$
h(\mathbf{p}, \mathbf{n}, \sigma)=2^{-1}\left(\mathbf{p}^{2}-\sigma\left(\mathbf{n}^{2}-\gamma\right)\right)
$$

и первичной связью $\varphi_{0}=\pi$. Уравнения движения принимают вид

$$
\begin{aligned}
\dot{f} & =\{h, f\}+\nu\left\{\varphi_{0}, f\right\}, \\
\varphi_{0} & =0 .
\end{aligned}
$$

Условие их совместности приводит к уравнению вторичной связи (аналогом матрищы $\gamma_{\nu \mu}$ здесь является $(1 \times 1)$ - “матрица" $\left.\left\{\varphi_{0}, \varphi_{0}\right\}=0\right)$

$$
0=\dot{\varphi}_{0}=\left\{h, \varphi_{0}\right\}=\varphi_{1} \equiv 2^{-1}\left(\mathbf{n}^{2}-\gamma\right)
$$

Условие совместности (22) и первого из уравнений движения (21) приводит к уравнению третичной связи

$$
0=\dot{\varphi}_{1}=\left\{h, \varphi_{1}\right\}=\varphi_{2} \equiv \mathbf{p n}
$$


(аналогом матрицы $\underset{\gamma}{\gamma}$ будет $\left\{\varphi_{0}, \varphi_{1}\right\}=0$ ). Та же картина возникает на следуюшем этапе "размножения" связей,

$$
0=\dot{\varphi}_{2}=\left\{h, \varphi_{2}\right\}=\varphi_{3} \equiv \mathbf{p}^{2}+\sigma \mathbf{n}^{2} .
$$

Наконец, последнее условие совместности

$$
0=\dot{\varphi}_{3}=\left\{h, \varphi_{3}\right\}+\nu\left\{\varphi_{0}, \varphi_{3}\right\}=4 \sigma \varphi_{2}+\nu \mathbf{n}^{2}
$$

фиксирует множитель Лагранжа $\nu$.

В нашем примере связи второго рода идентифицируются как таковые лишь на четвертом этапе "размножения": $\left\{\varphi_{0}, \varphi_{3}\right\}=\mathbf{n}^{2}$. Это связано с тем, что функция Гамильтона линейна по обобшенной координате $\sigma$, игравшей в первоначальной лагранжевой формулировке роль множителя Лагранжа, фиксируюшего векторное поле $\mathbf{n}$ на сфере $S^{n-1}$. Гессиан был вырожден именно по соответствующей скорости $\nu$, играющей роль множителя Лагранжа в гамильтоновой формулировке (19). Получающееся после его фиксации с помощью (25) дифференшиальное уравнение движения

$$
\dot{f}=\{h, f\}-4 \sigma \varphi_{2}\left(\mathbf{n}^{2}\right)^{-1}\left\{\varphi_{0}, f\right\}
$$

с учетом уравнения (23) третичной связи эквивалентно простейшему уравнению

$$
\dot{f}=\{h, f\},
$$

так что в нашем примере никакой скобки Дирака на этом этапе вводить не нужно.

Тем не менее редукция на поверхность связей $\varphi_{0}, \varphi_{3}$ имеет смысл. В этой паре связей разумно модифицировать (в духе процедуры раздела 3 ) вторую из них; ряд (12) для нее обрывается на первом члене, $\tilde{\varphi}_{3}=\sigma+\left(\mathbf{p}^{2} / \mathbf{n}^{2}\right)$. Возьмем после этого пару $\varphi_{0}, \tilde{\varphi}_{3}$ в качестве новых импульса и координаты для готовяшегося канонического преобразования (конечно, они изначально связаны с $\pi$ и $\sigma$ ). Подправка остальных канонических переменных элементарна: поскольку $\mathbf{p}$ и $\mathbf{n}$ имеют нулевые скобки Пуассона с $\varphi_{0}$, а выражения $\tilde{\mathbf{p}}$ и $\tilde{\mathbf{n}}$, обеспечиваюшие равенство нулю их скобок Пуассона с $\varphi_{3}-$ это ряды по степеням $\varphi_{0}$ с нулевыми членами $\mathbf{p}$ и $\mathbf{n}$, соответственно, то на поверхности $M_{0,3}=\left\{\varphi_{0}=0=\varphi_{3}\right\}$ каноническими переменными останутся $\mathbf{p}$ и $\mathbf{n}$.

Изменится на этой поверхности вид функции Гамильтона, поскольку в ней необходимо учесть уравнение связи $\varphi_{3}$,

$$
h_{(0)}=\left.h\right|_{M_{0,3}}=\mathbf{p}^{2}\left(1-\gamma\left(2 \mathbf{n}^{2}\right)^{-1}\right)=\mathbf{p}^{2} \frac{4 \varphi_{1}+\gamma}{2\left(2 \varphi_{1}+\gamma\right)} .
$$

Сужением на поверхность $M_{0,3}$ первоначальных уравнений движения и их следствий условий согласованности - оказывается система

$$
\dot{f}=\left\{h_{0}, f\right\}=0, \quad \varphi_{1}=0, \quad \varphi_{2}=0,
$$


причем связи $\varphi_{1}$ и $\varphi_{2}-$ второго рода: $\left\{\varphi_{2}, \varphi_{1}\right\}=\mathbf{n}^{2}$. В этой ситуации уравнения (29) могут быть интерпретированы как уравнения Эйлера-Лагранжа (и их следствия в форме условий совместности) для действия

$$
S[\mathbf{n} ; \mathbf{p} ; \lambda]=\int_{t_{1}}^{t_{2}} d t\left(-h_{0}-\lambda \varphi_{1}+\mathbf{p} \dot{\mathbf{n}}\right) .
$$

Эта гамильтонова форма не имеет лагранжева аналога: множитель Лагранжа $\lambda$ не является динамической переменной, поскольку не входит в 1-форму Картана-Лиувилля $\mathbf{p} d \mathbf{n}=\mathbf{p} \dot{\mathbf{n}} d t$.

Строго говоря, получающиеся из действия (30) уравнения Эйлера-Лагранжа имеют вид не (29), а

$$
\dot{f}=\left\{h_{0}, f\right\}+\lambda\left\{\varphi_{1}, f\right\}=0, \quad \varphi_{1}=0,
$$

уравнение связи $\varphi_{1}=0$ получается варьированием по $\lambda$ (при этом $\varphi_{1}-$ первичная связь в новой формулировке), а третье из уравнений (29) эквивалентно условию совместности

$$
0=\dot{\varphi}_{1}=\left\{h_{0}, \varphi_{1}\right\}=\varphi_{2} \equiv \mathbf{p n}\left(1-\gamma\left(2 \mathbf{n}^{2}\right)^{-1}\right)=\mathbf{p n} \frac{4 \varphi_{1}+\gamma}{2\left(2 \varphi_{1}+\gamma\right)}
$$

(мы сохраняем обозначение $\varphi_{2}$ для выражения, отличающегося ненулевым множителем от старого (23)). Наконец, последнее условие совместности

$$
0=\dot{\varphi}_{2}=\left\{h_{0}, \varphi_{2}\right\}+\lambda\left\{\varphi_{1}, \varphi_{2}\right\}=\mathbf{p n} \frac{2 \varphi_{1}+\gamma}{2 \varphi_{1}+2 \gamma}
$$

фиксирует множитель Лагранжа $\lambda$. Как и в случае с $\nu$, он оказывается пропорциональным связям, никакой модификации скобки Пуассона не происходит, и редукция на этом шаге сводится к построению канонического преобразования.

Эквивалентные паре $\varphi_{1}$ и $\varphi_{2}$ связи с единичной скобкой Пуассона, очевидно, имеют вид $\tilde{\varphi}_{1}=\varphi_{1}$ и $\tilde{\varphi}_{2}=\varphi_{2} /\left(2 \varphi_{1}+\gamma\right)$. Возьмем их в качестве пары канонических переменных, заменяющих последние компоненты векторов $\mathbf{n}$ и $\mathbf{p}$.

\section{СВОЙСТВА ПСЕВДОДИФФЕРЕНЦИАЛЬНЫХ ОПЕРАТОРОВ \\ ПРИЛОЖКНИЕ}

Воспользуемся соответствием между скобками Пуассона и векторными полями, отвечаюшими дифференциалам функций. Введем обозначения

$$
\{a,\{b\{c,\{\ldots, d\} \ldots\}\}\}=[a b c \ldots d], \quad[\underbrace{a a \ldots a}_{n} b]=\left[(a)_{n} b\right], \quad[a]=a .
$$

Тогда $Y^{\xi} f=\sum_{n \geqslant 0}(n !)^{-1}(-\eta)^{n}\left[(\xi)_{n} f\right]$ и по индукции легко доказывается, что

$$
\left[(\xi)_{n}(a b)\right]=\sum_{i=0}^{n} \frac{n !}{i !(n-i) !}\left[(\xi)_{i} a\right]\left[(\xi)_{n-i} b\right]
$$


Поскольку $\sum_{n \geqslant 0} \sum_{i=0}^{n}=\sum_{i \geqslant 0} \sum_{\nu \geqslant 0}$, где $\nu=n-i$, то из (П.2) следует, что

$$
Y^{(\xi)}(a b)=\sum_{n \geqslant 0}(n !)^{-1}(-\eta)^{n} \sum_{i=0}^{n} \frac{n !}{i !(n-i) !}\left[(\xi)_{i} a\right]\left[(\xi)_{n-i} b\right]=Y^{(\xi)} a \cdot Y^{\xi} b .
$$

Далее по индукции будем иметь

$$
\left[(\xi)_{n} a b\right]=\sum_{i=0}^{n} \frac{n !}{i !(n-i) !}\left\{\left[(\xi)_{i} a\right],\left[(\xi)_{n-i} b\right]\right\} .
$$

С помощью формулы (П.4) получим

$$
\begin{aligned}
Y^{(\xi)}\{a, b\}= & \sum_{n \geqslant 0}(n !)^{-1}(-\eta)^{n} \sum_{i=0}^{n} \frac{n !}{i !(n-i) !}\left\{\left[(\xi)_{i} a\right],\left[(\xi)_{n-i} b\right]\right\}= \\
= & \left\{Y^{(\xi)} a, Y^{(\xi)} b\right\}-\sum_{i \geqslant 0, \nu \geqslant 0}(i !)^{-1}((\nu-1) !)^{-1}(-\eta)^{i+\nu-1}\left[\eta(\xi)_{i} a\right] \cdot\left[(\xi)_{\nu} b\right]- \\
& -\sum_{i \geqslant 1, \nu \geqslant 0}(\nu !)^{-1}((i-1) !)^{-1}(-\eta)^{i+\nu-1}\left[\eta(\xi)_{i} b\right] \cdot\left[(\xi)_{\nu} a\right]= \\
= & \left\{Y^{(\xi)} a, Y^{(\xi)} b\right\}-Y^{(\xi)}\{\xi, a\} \cdot Y^{(\xi)}\{\eta, b\}-Y^{(\xi)}\{\eta, a\} \cdot Y^{(\xi)}\{\xi, b\},
\end{aligned}
$$

где учтено, что

$$
\xi \eta\{a b\}=\{\xi a, \eta b\}+\xi b\{\eta, a\}-\eta a\{\xi, b\}-a b .
$$

Окончательно находим

$$
\left\{Y^{(\xi)} a, Y^{(\xi)} b\right\}=Y^{(\xi)}(\{a, b\}-\{a, \xi\}\{\eta, b\}+\{a, \eta\}\{\xi, b\})=Y^{(\xi)}\{a, b\}_{\xi \eta}^{\mathrm{D}} .
$$

Поскольку псевдодифференциальные операторы, относящиеся к связям, коммутируют, формулы (П.3) и (П.5) остаются в силе после применения к ним любого числа операторов и в любом их сочетании. Наконец, полезно указать формулу, обратную к (13),

$$
\left\{\xi, \int_{0}^{\eta} d \eta Y^{(\xi)} a\right\}=a .
$$

\section{Список литературы}

[1] В. П. Павлов. ТМФ. 1992. Т. 92. № 3. С. 451-456.

[2] В. П. Павлов, А. О. Старинец. ТМФ. 1995. Т. 105. № 3. С. 429-437.

[3] В. П. Павлов. Тр. МИАН. 2000. Т. 228. С. 145-154.

[4] Д. М. Гитман, И. В. Тютин. Каноническое квантование систем со связями. М.: Наука, 1986.

[5] Л. Д. Фаддеев. ТМФ. 1969. Т. 1. № 1. С. 3-18.

[6] Ф. Р. Гантмахер. Теория матриц. М.: Наука, 1966. 